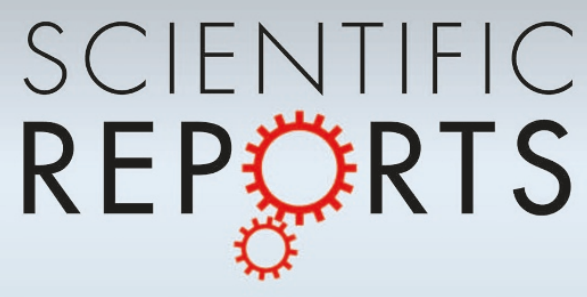

OPEN

SUBJECT AREAS:

BIOCHEMISTRY

ENVIRONMENTAL SCIENCES

BIOMATERIALS

Received

3 February 2014

Accepted

19 March 2014

Published

8 April 2014

Correspondence and requests for materials should be addressed to C.-S.Z. (zhaochsh70@ scu.edu.cn or zhaochsh70@163.

com)

\section{From Commodity Polymers to Functional Polymers}

\author{
Tao Xiang ', Ling-Ren Wang' ', Lang Ma' , Zhi-Yuan Han' ', Rui Wang' ', Chong Cheng' ', Yi Xia', Hui Qin' \\ \& Chang-Sheng Zhao ${ }^{1,2}$
}

${ }^{1}$ College of Polymer Science and Engineering, State Key Laboratory of Polymer Materials Engineering, Sichuan University, Chengdu 610065, People's Republic of China, ${ }^{2}$ National Engineering Research Center for Biomaterials, Sichuan University, Chengdu 610064, China.

Functional polymers bear specified chemical groups, and have specified physical, chemical, biological, pharmacological, or other uses. To adjust the properties while keeping material usage low, a method for direct synthesis of functional polymers is indispensable. Here we show that various functional polymers can be synthesized by in situ cross-linked polymerization/copolymerization. We demonstrate that the polymers synthesized by the facile method using different functional monomers own outstanding $\mathrm{pH}$-sensitivity and $\mathrm{pH}$-reversibility, antifouling property, antibacterial, and anticoagulant property. Our study opens a route for the functionalization of commodity polymers, which lead to important advances in polymeric materials applications.

F unctional polymers are macromolecules that have specific properties or uses ${ }^{1,2}$. The properties of such materials are often determined by the presence of chemical functional groups that are dissimilar to those of the backbone chains. Functional polymers can be synthesized by direct anionic, cationic, free radical and coordination polymerization, as well as the bulk and surface modification of preformed polymer backbones ${ }^{2}$. To adjust the properties while keeping material usage low, a simple method for direct synthesis of functional polymers using commodity polymers and functional monomers is indispensable. Here we provide a facile way for the synthesis of functional polymers by in situ cross-linked polymerization/copolymerization in commodity polymer solutions based on recent studies, ${ }^{3,4}$, as shown in Scheme S1. Several commodity polymers, including polyethersulfone (PES), polysulfone (PSf), polyvinylidene fluoride (PVDF) and polystyrene (PS) are selected for the functionalization; and many vinyl functional monomers, including acrylic acid (AA), 4-vinyl pyridine (4-VP), sulfobetaine methacrylate (SBMA), methacrylatoethyl trimethyl ammonium chloride (DMC), N-vinylpyrrolidone (NVP), and sodium p-styrene sulfonate (NaSS) are selected in situ cross-linked polymerization/copolymerization. Then, stimuli-responsive, antifouling, antibacterial and blood compatible polymers are obtained. The polymer solutions can be directly prepared into membranes by a liquid-liquid phase separation technique ${ }^{5}$.

\section{Results}

Stimuli-responsive polymers. Stimuli-responsive ("intelligent") materials and membranes exhibit abrupt property changes in response to small changes in external stimuli such as temperature, $\mathrm{pH}$, ionic-strength and/or solvent composition of the media, concentration of specific chemical species, the electric field, and photo-irradiation ${ }^{6,7,89}$. Compared with other external stimuli, $\mathrm{pH}$-sensitivity gives more choices both for the materials and for the application environment, making it a powerful technique. Herein, we firstly prepared $\mathrm{pH}-$ sensitive polymers by in situ cross-linked polymerization of acrylic acid (AA) and 4-vinyl pyridine (4-VP) in PES and PVDF solutions, respectively. The polymer solutions are directly prepared into porous membranes by a liquid-liquid phase separation technique, and the membranes are characterized by ATR-FTIR, ${ }^{1} \mathrm{H}$ NMR and SEM, as shown in Fig. S1, Fig. S2 and Fig. S3 (see Supporting Information, SI). Thus, the target functional polymers were successfully synthesized according to the characterization.

Fig. 1 shows the $\mathrm{pH}$-sensitivity and $\mathrm{pH}$-reversibility of the as-prepared PES membranes. The flux for the PES membrane modified by the cross-linked polymerization of AA exhibits a chemical valve behavior at $\mathrm{pH}$ between 7.0 and 9.0, and hardly changed at the $\mathrm{pH}$ values lower than 7.0 or higher than 9.0. After adding methyl methacrylate (MMA) into the polymerization solution as the hydrophobic monomer, the chemical valve behavior appears at the $\mathrm{pH}$ between 4.0 and 7.0. The results indicated that the chemical valve behavior could be adjusted by copolymerization. The opposite changing trend of water flux for PSf/P4-VP was observed as shown in Fig. S3. 

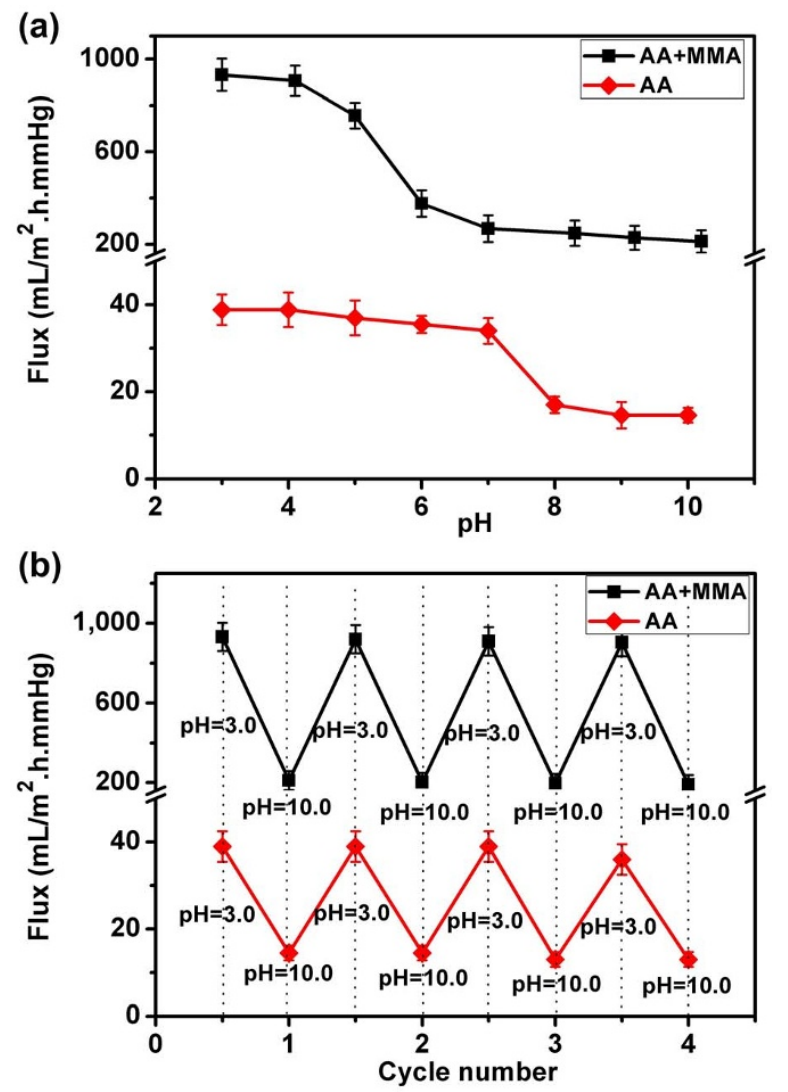

Figure $1 \mid$ (a). Water flux for the membrane as a function of $\mathrm{pH}$ values. (b). Water flux for the membranes as the feed solutions are changed between $\mathrm{pH} 3.0$ and $\mathrm{pH} 10.0$ with 10 min equilibration flow followed by $10 \mathrm{~min}$ sample collection. For membranes: $\mathrm{PES} / \mathrm{P}(\mathrm{AA}-\mathrm{MMA})(\boldsymbol{\square})$; $\mathrm{PES} / \mathrm{PAA}(\boldsymbol{\nabla})$. Duplicate experiments showed similar results.

Antifouling polymers. Fouling is the accumulation of unwanted molecules on solid surfaces to the detriment of function, such as membrane fouling ${ }^{10,11}$. Membrane fouling is the deposition of retained particles, colloids, macromolecules, salts, etc, at the membrane surface or inside the pore at the pore wall, which causes a higher energy use, a higher cleaning frequency and a shorter life span of the membrane ${ }^{11}$. Recently, zwitterionic polymers had been widely investigated to improve the antifouling and biocompatibility of materials ${ }^{12,13,14,15}$. Among them, poly(2-methacryloyloxyethyl phosphorylcholine) (PMPC), poly(sulfobetaine methacrylate) (PSBMA) and poly(carboxybetaine methacrylate) (PCBMA) were widely used. Herein, we prepared antifouling polymer by in situ cross-linked polymerization of sulfobetaine methacrylate (SBMA) in PES solution. Then the polymer solution was directly prepared into porous membrane. Thermogravimetric analysis (TGA) was used to investigate the chemical compositions of the modified membranes as shown in Fig. S4. It showed that the contents of PSBMA in the modified membranes were almost the same as the corresponding theoretical contents, indicating that the conversion ratios of the SBMA were nearly $100 \%$ and there was no elution of PSBMA in the membrane preparation process. The antifouling property of the membranes was evaluated by the ultrafiltration of bovine serum albumin (BSA) solution, and the fluxes of BSA solution and $\mathrm{PBS}$ for three-cycle filtrations are shown in Fig. 2. It showed that the flux recovery ratios $\left(\left(\mathrm{F}_{\mathrm{RR}} \mathrm{s}\right)\right)$ of the membranes prepared by PSBMA with the amount above $4 w t$. \% in the casting solution are nearly $100 \%$, indicating that the PSBMA modified membranes possessed excellent antifouling property and the membranes could be used in complex media.

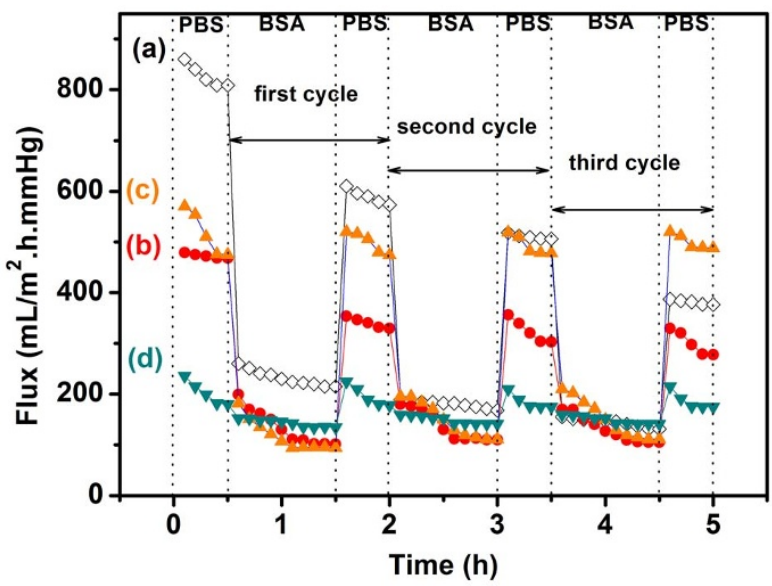

Figure $2 \mid$ Time-dependent fluxes during a process of three-cycle of BSA ultrafiltration at room temperature for membranes PES (a, $\diamond)$, PES/ PSBMA-2\% (b, ๑), PES/PSBMA-4\% (c, $\triangle$ ) and PES/PSBMA-6\% (d, $\nabla$ ). Each recycle includes the ultrafiltration of BSA solution $(1.0 \mathrm{mg} / \mathrm{mL})$ and then PBS.

Antibacterial polymers. Recently, tethering of nontoxic functional polymer brush coatings has been suggested as an environmentally friendly approach to prevent the attachment of proteins, bacteria, and marine organisms to surfaces ${ }^{16}$. Quaternary ammonium cations form a class of compounds which are used as biocides and corrosion inhibitors ${ }^{17}$. As biocides, quaternary ammonium cations act on the plasmic membranes of cells, disrupt the lipid bilayers, and cause the release of intracellular materials ${ }^{18}$. The surfactant property of these compounds provides additional protection against the formation of polysaccharidic materials during the process of bacteria colonization $^{19}$. Vinyl monomers containing tertiary amino groups, such as 2-(dimethylamino) ethyl methacrylate (DMAEMA) and 4vinyl pyridine (4VP), have been extensively used in the synthesis of antimicrobial surfaces ${ }^{20,21}$. In this study, antibacterial polymers of poly(methacrylatoethyl trimethyl ammonium chloride) (PDMC) were prepared by in situ cross-linked polymerization of DMC in PSf solution and PS solution, respectively. The antibacterial efficiency of the PDMC modified PSf/PDMC and PS/PDMC membranes toward both gram-positive Staphylococcus aureus (S. aureus) and gram-negative Escherichia coli (E. coli) bacteria were studied. The SEM images are shown in the Fig. S6, for the membrane surfaces after bacterial fouling, a large amount of

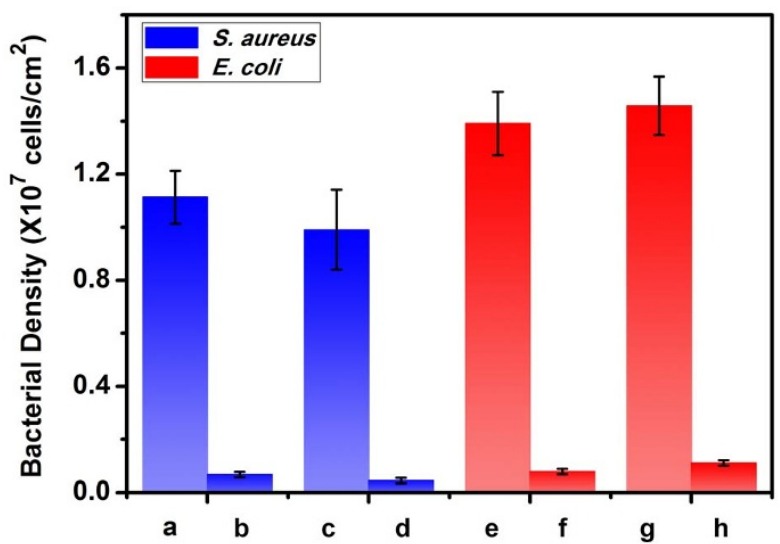

Figure 3 Bacterial cell density of $S$. aureus and $E$. coli on the pristine and modified membranes (a) PSf, (b) PSf/PDMC, (c) PS, (d) PS/PDMC after exposuring to $S$. aureus $\left(10^{6}\right.$ cells/mL) for $24 \mathrm{~h}$; (e) PSf, (f) PSf/PDMC, (g) PS, (h) PS/PDMC after exposuring to E. coli $\left(10^{6}\right.$ cells $\left./ \mathrm{mL}\right)$ for $24 \mathrm{~h}$. 


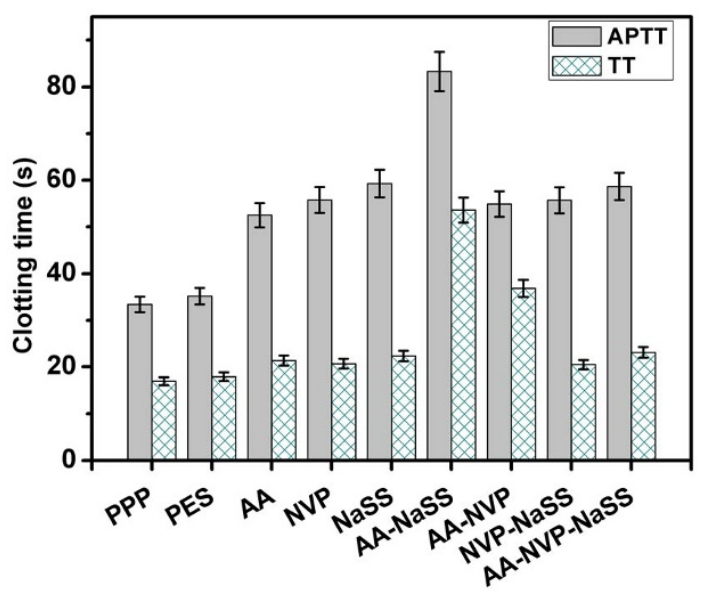

Figure $4 \mid$ Activated partial thromboplastin times (APTTs) and thrombin times (TTs) for the membranes modified by PAA, PNVP, $\mathrm{PNaSS}$ and the copolymers. Values are expressed as means $\pm \mathrm{SD}, \mathrm{n}=3$.

bacteria adhered on the surface of the pristine surface. After that PDMC was introduced into the membranes, the amount of the adhered bacteria was obviously reduced. In addition, the traditional surface plating method was used to quantify the antibacterial property of the PDMC membranes, and the results are showed in Fig. 3. It was found that the amounts of the adhered $S$. aureus and E. coli bacteria were only about $8 \%$ of those of the pristine PSf and PS membranes. The results indicated that the antibacterial property of the PDMC modified membranes was obviously improved.

Blood compatible polymers. When blood is in contact with a biomaterial, plasma protein adsorption on the surface is the first event, followed by platelet adhesion and activation of coagulation pathways, and then leading to thrombus formation ${ }^{22}$. To improve the blood compatibility, the current trend is to develop new materials and structures. However, alternative materials often prove more vulnerable in terms of stability and are often expensive; a good method is the modification of common polymers or membranes. There are three main approaches for the modification of polymeric membranes with improved blood compatibility ${ }^{23}$ : (1) bulk modification of polymeric material, and then to prepare modified membrane; (2) surface modification of prepared membrane; and (3) blending, which can also be regarded as a surface modification. Herein, the facile in situ cross-linked polymerization is used for the modification of PES membrane using different monomers of AA, VP, and NaSS with the same weight ratios. The anticoagulant property of the membranes was evaluated by activated partial thromboplastin time (APTT) and thrombin time (TT) as shown in
Fig. 4. The APTTs were obviously increased for the modified membranes. Furthermore, the membranes modified by AA and NaSS showed significantly improved anticoagulant property (as expressed by prolonged APTT and TT) due to the heparin-like structure $e^{24,25,26}$.

\section{Discussion}

We applied the in situ cross-linked polymerization/copolymerization method to adjust the properties of commodity polymers while keeping material usage low. The commodity polymers including PES, PSf, PVDF and PS were used to be functionalized with stimuli-responsive, antifouling, antibacterial and blood compatible properties by introducing PAA and P4VP, PSBMA, PDMC, PNVP and PNaSS, respectively. The advantages of our method includes (as shown in Table S1): (1). the post-processing is simple, without precipitation and dialysis; (2). the reaction solutions can be directly prepared into membranes after being cooled and degassed; (3). the miscibility between the commodity polymers and functional polymers synthesized by in situ cross-linked polymerization/copolymerization method is improved compared with conventional simple blend method; (4). the structure of the materials prepared by in situ cross-linked polymerization/copolymerization method was stable since cross-linkers were added into the reaction solution with the monomers. Thus, our study opens a practical, simple, and low-cost route for the functionalization of commodity polymers, which lead to important advances in polymeric materials applications, such as separation fields and biomedical fields.

\section{Methods}

Materials. Polyethersulfone (PES, Ultrason E6020P, BASF), polysulfone (PSf, Udel P-1700, Union Carbide), polyvinylidene fluoride (PVDF, Xiya Reagent) and polystyrene (PS, Xiya Reagent) were the representative commodity polymers for the modification. Acrylic acid (AA, 99\%, Aladdin), 4-vinyl pyridine (4-VP, 96\%, Aladdin) and N-vinylpyrrolidone (NVP, 99\%, Aladdin) were distilled under reduced pressure to remove the inhibitor. Methacrylatoethyl trimethyl ammonium chloride (DMC, 75 wt. \% in $\mathrm{H}_{2} \mathrm{O}$, Aladdin) and sodium p-styrene sulfonate (NaSS, $90 \%$, Aladdin) were used as received. Sulfobetaine methacrylate (SBMA) was synthesized according to our previous reported procedures ${ }^{27}$. Micro $\mathrm{BCA}^{\mathrm{TM}}$ protein assay reagent kits were the products of PIERCE. APTT and TT reagent kits were purchased from SIEMENS. Dimethyl sulfoxide (DMSO, 99.8\%, Kelong) and N,N-dimethylacetamide (DMAc, 99.8\%, Kelong) were used without further purification. Deionized water was used throughout the studies.

Preparation of functional membranes via in situ cross-linked polymerization. In this study, various commodity polymers were endowed with desired functions via in situ cross-linked polymerization, including $\mathrm{pH}$-sensitivity and $\mathrm{pH}$-reversibility, antifouling property, antibacterial, and anticoagulant property (Table 1). To prepare functional membranes, one kind of commodity polymer was completely dissolved in the good solvent, such as DMAc and DMSO. Then, the monomers, initiator and cross-linker mixture was added into the polymer solution and the reaction time was long enough to ensure the percent conversion of the monomers. A typical procedure to prepare $\mathrm{pH}$-sensitive membrane was as follows: $16 \mathrm{~g}$ PES (16 wt. \% of the total solution) was dissolved in DMAc to get a homogeneous solution. Then a mixture of monomers, AIBN ( $1 \sim 4$ mol\% with respect to monomers), MBA $(0.5 \sim 2 \mathrm{~mol} \%$

Table 1 | The compositions for the in situ cross-linked polymerization/copolymerization

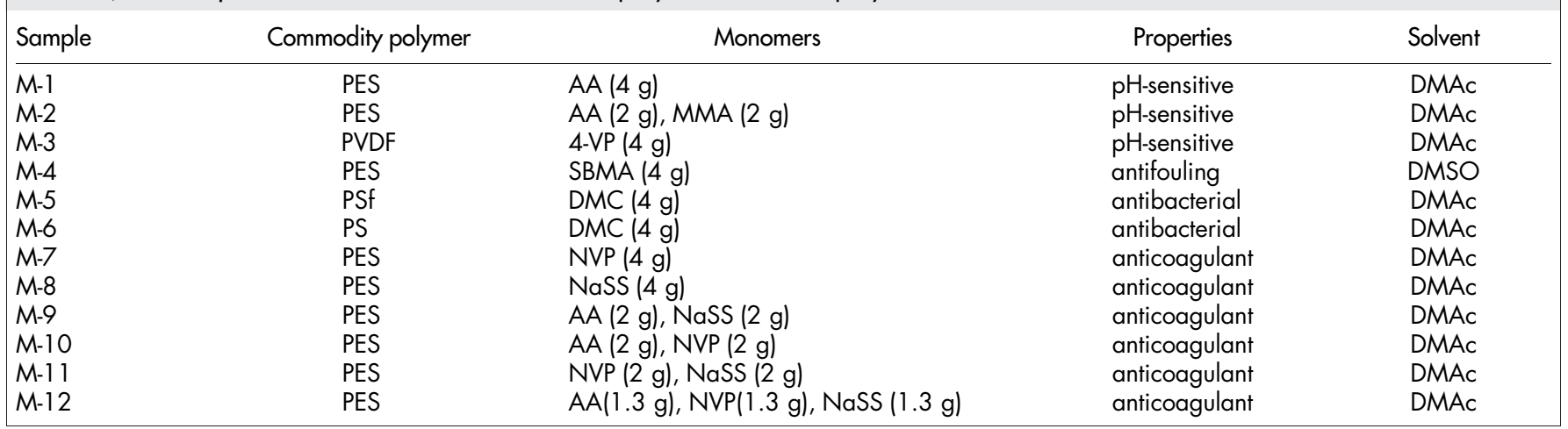


with respect to monomers) and DMAc was added in the PES solution under nitrogen atmosphere. The polymerization was carried out at $75^{\circ} \mathrm{C}$ with vigorous stirring for about $24 \mathrm{~h}$. The cross-linked density depends on the molecular weights of the monomers and the matrix polymers. In this study, the cross-linked density was estimated by the contents of the monomers and the cross-linking agent in the polymerization, and which was ranged from 3600 to $14000 \mathrm{~g} / \mathrm{mol}$ (details were shown in SI).

After that the reaction was completed, the polymer solution was directly prepared into membrane by a liquid-liquid phase separation technique after vacuum degassing. The casting solution was added on a glass plate by spin-coating and then the glass was immersed into deionized water, which was used as a nonsolvent. The solvent could exchange with deionized water and the functional membrane was then prepared. Since the synthesized functional polymer was cross-linked and the commodity polymer did not participate in the polymerization reaction, semi-interpenetrating network (semi-IPN) membrane was obtained.

Characterizations. Attenuated total reflection-Fourier transform infrared spectroscopy (ATR-FTIR) was obtained on a Nicolet 560 spectrophotometer (Nicol, America) between 4000 and $675 \mathrm{~cm}^{-1}$, with a resolution of $4 \mathrm{~cm}^{-1}$.

The cross-section morphology of the membranes was observed by using JSM$7500 \mathrm{~F}$ field-emission scanning microscope (SEM) (JEOL, Japan) with the voltage of $5 \mathrm{kV}$. The membrane samples were dried overnight in a vacuum oven at room temperature, then quenched by liquid nitrogenous gas, attached to the sample supports and coated with a gold layer.

Thermogravimetric analysis (TGA) was performed on a TG209F1 TG instrument (Netzsch, Germany) at a heating rate of $10^{\circ} \mathrm{C} / \mathrm{min}$ under $\mathrm{N}_{2}$ atmosphere. The samples were dried at $110^{\circ} \mathrm{C}$ to remove any moisture prior to the study.

Ultrafiltration experiments. The $\mathrm{pH}$-sensitivity and $\mathrm{pH}$-reversibility properties of the modified membranes were evaluated through measuring the water flux by varying the $\mathrm{pH}$ of the feed solution with $\mathrm{NaOH}$ or $\mathrm{HCl}$, by using the apparatus as described in our previous study ${ }^{28}$. The test membranes were pre-compacted with pure water for 30 min to get steady filtration. Then, the water flux was determined at the $\mathrm{pH}$ values ranging from 3.0 to 10.0 , randomly. For the $\mathrm{pH}$-reversibility experiment, the feed solution was alternatively changed between 3.0 and 10.0. The flux was calculated by using the following equation:

$$
F l u x=\frac{V}{S P t}
$$

where $V$ is the volume of the permeated solution $(\mathrm{mL}) ; S$ is the effective membrane area $\left(\mathrm{m}^{2}\right) ; P$ is the pressure applied to the membrane $(\mathrm{mmHg})$ and $t$ is the time $(\mathrm{h})$.

The BSA solution ( $1 \mathrm{mg} / \mathrm{mL}$ in PBS) was applied to estimate the antifouling property of the modified membranes, and the protein flux was measured as above. The BSA rejection ratio $(R)$ was defined as follows:

$$
R(\%)=\left(1-\frac{C_{p}}{C_{b}}\right) \times 100
$$

where $\mathrm{C}_{p}$ and $\mathrm{C}_{b}(\mathrm{mg} / \mathrm{mL})$ are the BSA concentrations of the permeated and bulk solutions, respectively, which are measured by an UV-vis spectrophotometer (UV1750, Shimadzu, Japan) at the wavelength of $278 \mathrm{~nm}$. After protein filtration, the membrane was cleaned with deionized water; the PBS flux of the cleaned membrane was measured again and the flux recovery ratio $\left(F_{R R}\right)$ was calculated using the following equation:

$$
F_{R R}(\%)=\left(\frac{F_{2}}{F_{1}}\right) \times 100
$$

where $F_{1}$ and $F_{2}\left(\mathrm{~mL} / \mathrm{m}^{2} \cdot \mathrm{h} \cdot \mathrm{mmHg}\right)$ are the PBS flux before and after protein ultrafiltration, respectively.

Antibacterial measurement. Gram-positive Staphylococcus aureus (S. aureus) and gram-negative Escherichia coli (E. coli) are used to evaluate the antibacterial adhesion characteristics and bactericidal efficacy of the modified membranes. After incubation in beef extract peptone medium (BPDA), bacterial cells were suspended at a concentration of $10^{6}$ cells $/ \mathrm{mL}$. Each piece of membrane was then immersed in $1 \mathrm{~mL}$ of bacterial suspension under static condition at $37^{\circ} \mathrm{C}$ for $24 \mathrm{~h}$. The adhered bacterial cells were investigated under a scanning electron microscope (SEM) (JSM-7500F, JEOL, Japan) after fixing with $3 \%$ glutaraldehyde and dehydration with serial ethanol solutions $(25 \%, 50 \%, 75 \%$ and $100 \%)$. To quantify the antibacterial property of the PDMC membranes, the membranes were rinsed three times with PBS after being immersed in bacterial suspension under static condition at $37^{\circ} \mathrm{C}$ for $24 \mathrm{~h}$, and then immersed in BPDA under ultrasound to make sure the adhered bacterial cells were suspended in the BPDA. The suspension was diluted and $0.1 \mathrm{~mL}$ of the diluted suspension was spread onto a triplicate solid agar plate. The plate was then sealed and incubated at $37^{\circ} \mathrm{C}$ for $24 \mathrm{~h}$ and the number of the viable cells was counted.

Clotting time. To evaluate the antithrombogenicity of the modified membranes, activated partial thromboplastin time (APTT) and thromboplastin time (TT) were measured by a semiautomatic blood coagulation analyzer CA-50 (Sysmex Corporation, Kobe, Japan).
The APTT test method was performed as follows. The blood was centrifuged at $1000 \mathrm{rpm}$ for $15 \mathrm{~min}$ to obtain platelet-rich plasma (PRP) or at $4000 \mathrm{rpm}$ for $15 \mathrm{~min}$ to obtain platelet-poor plasma (PPP). The membrane $\left(0.5 \times 0.5 \mathrm{~cm}^{2}\right.$, three pieces) was immersed in $0.2 \mathrm{ml} \mathrm{PBS}$ buffer ( $\mathrm{pH} 7.4)$ for $1 \mathrm{~h}$. Then, the PBS was removed, and $0.1 \mathrm{~mL}$ of fresh PPP was introduced. After incubating at $37^{\circ} \mathrm{C}$ for $30 \mathrm{~min}, 50 \mu \mathrm{L}$ of the incubated PPP was added into the test cup, followed by the addition of $50 \mu \mathrm{L}$ of APTT agent (Dade Actin Activated Cephaloplastin Reagent, Siemens; incubated $10 \mathrm{~min}$ before use). Thereafter, $50 \mu \mathrm{L}$ of $0.025 \mathrm{M} \mathrm{CaCl}_{2}$ solution was added, and then the APTT was measured. For TT test, $50 \mu \mathrm{L}$ incubated PPP was mixed well with $100 \mu \mathrm{L}$ of TT agent (Thromborel ${ }^{\circledR}$ S, Siemens) at $37^{\circ} \mathrm{C}$ for $2 \mathrm{~min}$, and the TT was measured. At least three measurements were averaged to get a reliable value.

1. Bergbeiter, D. E. \& Martin, C. R. Functional Polymers (Plenum Press, New York, 1989).

2. Schulz, D. N. \& Patil, A. O. Functional Polymers: An overview (ACS, Washington, DC, 1998).

3. Zhang, P. Y., Xu, Z. L., Yang, H., Wei, Y. M. \& Wu, W. Z. Fabrication and characterization of PVDF membranes via an in situ free radical polymerization method. Chem. Eng. Sci. 97, 296-308 (2013).

4. Tao, M. M., Liu, F. \& Xue, L. X. Hydrophilic poly (vinylidene fluoride)(PVDF) membrane by in situ polymerisation of 2-hydroxyethyl methacrylate (HEMA) and micro-phase separation. J. Mater. Chem. 22, 9131-9137 (2012).

5. Matsuyama, H., Nishiguchi, M. \& Kitamura, Y. Phase separation mechanism during membrane formation by dry-cast process. J. Appl. Polym. Sci. 77, 776-783 (2000).

6. Zhao, C. S., Nie, S. Q., Tang, M. \& Sun, S. D. Polymeric pH-sensitive membranesA review. Prog. Polym. Sci. 36, 1499-1520 (2011).

7. Thomas, J. B., Tingsanchali, J. H., Rosales, A. M., Creecy, C. M., McGinity, J. W. \& Peppas, N. A. Dynamics of poly (ethylene glycol)-tethered, $\mathrm{pH}$ responsive networks. Polymer 48, 5042-5048 (2007).

8. Dayananda, K., He, C., Park, D. K., Park, T. G. \& Lee, D. S. pH-and temperaturesensitive multiblock copolymer hydrogels composed of poly (ethylene glycol) and poly (amino urethane). Polymer 49, 4968-4973 (2008).

9. Gil, E. S. \& Hudson, S. M. Stimuli-reponsive polymers and their bioconjugates. Prog. Polym. Sci. 29, 1173-1222 (2004).

10. Yebra, D. M., Kiil, S. \& Dam-Johansen, K. Antifouling technology-past, present and future steps towards efficient and environmentally friendly antifouling coatings. Prog. Org. Coat. 50, 75-104 (2004).

11. Rana, D. \& Matsuura, T. Surface modifications for antifouling membranes. Chem. Rev. 110, 2448 (2010).

12. Huang, C. J., Li, Y. T. \& Jiang, S. Y. Zwitterionic Polymer-Based Platform with Two-Layer Architecture for Ultra Low Fouling and High Protein Loading. Anal. Chem. 84, 3440-3445 (2012).

13. Liu, P. S., Chen, Q., Wu, S. S., Shen, J. \& Lin, S. C. Surface modification of cellulose membranes with zwitterionic polymers for resistance to protein adsorption and platelet adhesion. J. Membr. Sci. 350, 387-394 (2010).

14. Ma, X. L., Su, Y. L., Sun, Q., Wang, Y. Q. \& Jiang, Z. Y. Preparation of proteinadsorption-resistant polyethersulfone ultrafiltration membranes through surface segregation of amphiphilic comb copolymer. J. Membr. Sci. 292, 116-124 (2007).

15. Chang, Y., Shih, Y. J., Lai, C. J., Kung, H. H. \& Jiang, S. Y. Blood-Inert Surfaces via Ion-Pair Anchoring of Zwitterionic Copolymer Brushes in Human Whole Blood. Adv. Funct. Mater. 23, 1100-1110 (2013).

16. Yang, W. J., Pranantyo, D., Neoh, K. G., Kang, E. T., Teo, S. L. M. \& Rittschof, D. Layer-by-Layer Click Deposition of Functional Polymer Coatings for Combating Marine Biofouling. Biomacromolecules 13, 2769-2780 (2012).

17. Neoh, K. \& Kang, E. Combating bacterial colonization on metals via polymer coatings: relevance to marine and medical applications. ACS Appl. Mater. Inter. 3, 2808-2819 (2011).

18. Tiller, J. C., Lee, S. B., Lewis, K. \& Klibanov, A. M. Polymer surfaces derivatized with poly (vinyl-N-hexylpyridinium) kill airborne and waterborne bacteria. Biotechnol. Bioeng. 79, 465-471 (2002).

19. Videla, H. A. Prevention and control of biocorrosion. Int. Biodeterior. Biodegrad. 49, 259-270 (2002)

20. Yuan, S. J., Pehkonen, S. O., Ting, Y. P., Neoh, K. G. \& Kang, E. T. Antibacterial Inorganic-Organic Hybrid Coatings on Stainless Steel via Consecutive SurfaceInitiated Atom Transfer Radical Polymerization for Biocorrosion Prevention. Langmuir 26, 6728-6736 (2009).

21. Huang, J. et al. Nonleaching antibacterial glass surfaces via "grafting onto": the effect of the number of quaternary ammonium groups on biocidal activity. Langmuir 24, 6785-6795 (2008).

22. Andrade, J. \& Hlady, V. Plasma Protein Adsorption: The Big Twelvea. Ann. N.Y. Acad. Sci. 516, 158-172 (1987).

23. Zhao, C. S., Xue, J. M., Ran, F. \& Sun, S. D. Modification of polyethersulfone membranes-A review of methods. Prog. Mater Sci. 58, 76-150 (2013).

24. Ran, F., Nie, S. Q., Li, J., Su, B. H., Sun, S. D. \& Zhao, C. S. Heparin-Like Macromolecules for the Modification of Anticoagulant Biomaterials. Macromol. Biosci. 12, 116-125 (2012).

25. Tang, M., Xue, J. M., Yan, K. L., Xiang, T., Sun, S. D. \& Zhao, C. S. Heparin-like surface modification of polyethersulfone membrane and its biocompatibility. J. Colloid Interface Sci. 386, 428-440 (2012). 
26. Wang, L. R., Qin, H., Nie, S. Q., Sun, S. D., Ran, F. \& Zhao, C. S. Direct synthesis of heparin-like poly (ether sulfone) polymer and its blood compatibility. Acta Biomater. 9, 8851-8863 (2013).

27. Yue, W. W., Xiang, T., Zhao, W. F., Sun, S. D. \& Zhao, C. S. Preparation and characterization of $\mathrm{pH}$-sensitive polyethersulfone membranes blended with poly (methyl methacrylate-co-maleic anhydride) copolymer. Sep. Sci. Technol. 43, 1941-1953 (2013)

28. Li, L. L., Yin, Z. H., Li, F. L., Xiang, T., Chen, Y. \& Zhao, C. S. Preparation and characterization of poly (acrylonitrile-acrylic acid-N-vinyl pyrrolidinone) terpolymer blended polyethersulfone membranes. J. Membr. Sci. 349, 56-64 (2010).

\section{Acknowledgments}

This work was supported by the National Natural Science Foundation of China (No. 51173119 and 51225303), and the Programme of Introducing Talents of Discipline to Universities (B13040). We should also thank our laboratory members for their generous help, and gratefully acknowledge the help of Ms. Hui. Wang.

\section{Author contributions}

T.X. and C.-S.Z. conceived and designed the study. C.-S.Z. supervised the research. T.X., L.-R.W. and L.M. performed membranes preparation and characterization experiments. Z.-Y.H., R.W., C.C., Y.X. and H.Q. performed ultrafiltration experiments, clotting time and antibacterial measurements. All authors discussed the results and contributed to the data interpretation. T.X. and C.-S.Z. wrote the manuscript and all authors commented on the manuscript.

\section{Additional information}

Supplementary information accompanies this paper at http://www.nature.com/ scientificreports

Competing financial interests: The authors declare no competing financial interests.

How to cite this article: Xiang, T. et al. From Commodity Polymers to Functional Polymers. Sci. Rep. 4, 4604; DOI:10.1038/srep04604 (2014).

(c) (1) (5) This work is licensed under a Creative Commons Attribution-NonCommercialcc. ${ }_{\mathrm{BY}} \mathrm{NC}_{\mathrm{SA}}$ ShareAlike 3.0 Unported License. The images in this article are included in the article's Creative Commons license, unless indicated otherwise in the image credit; if the image is not included under the Creative Commons license, users will need to obtain permission from the license holder in order to reproduce the image. To view a copy of this license, visit http://creativecommons.org/licenses/by-nc-sa/3.0/ 